\title{
Role of 2D-material in engineered nanomaterials
}

\section{Editorial}

With the advances in nanotechnology, we are now entering into a new era of 'engineered materials by deign' using fundamental building blocks that are derived from the inorganic, organic, polymeric, and biomolecular species. ${ }^{1,2}$ Further, materials are currently getting synthesized by invoking some additional processes over and above those of the conventional chemical reactions. These include sitespecific chemical conjugations comprising of covalent as well as weak chemical bonds along with the topology specific interactions of the molecular species. For example, usage of nanoparticulate material building blocks in syntheses are noted to facilitate self-limiting control of their organizational behaviors that enable them to very precisely control their final morphologies that impart characteristic features to different molecular complexes so realized. ${ }^{3}$ This includes controlled realization of different physico-chemico-biological properties quite close to those of the smart and intelligent behaviors of biological systems. Learning and employing biomimetic processes from the Nature are therefore proving to be of quite a great help in this quest. ${ }^{4}$

In this whole context of engineered materials, of late, the recent entry of two-dimensional layered/non layered materials have already started providing numerous alternatives for changing the materials designs with better controls in contrast to those of the nanoparticulate species just by restricting their quantum confinement in transverse directions alone. ${ }^{5}$ Out of such a large number of 2D-materials, explored so far, the ones comprising of monolayer forms are of special interest theoretically as well as experimentally. 1D-quantum confinement of their charge carriers are invariably found possessing very high mobility and saturation limited drift velocities combined with the absence of dangling bonds on either of the two sides imparting novel material properties arising out of self-organization of their corresponding unit cells that are held together strongly in a regular lattice with very little interlayer interactions (in case of layered 2D-materials). This kind of arrangement is free from most irritating phenomena of self-aggregation/coagulation observed in other systems comprising of NPs. Having recognized such novel features of 2D-materials in terms of wide variation in their energy band gaps (i.e. from zero to more than $6 \mathrm{eV}$ ), very high carrier mobility and saturation limited velocity in addition to their surface functionalization, quite aggressive efforts are now going on to increase the size of the mono/ multiple-layers for active device fabrications prior to their large scale industrial applications involving their integration for sub functional modules. ${ }^{5}$ Even preparation of nanocomposites of 2D-nanomaterials and polymeric compounds are currently being developed for their varied applications with added advantages not foreseen earlier., ${ }^{5,6}$ The role of ionic liquids has currently been found facilitating easier surface functionalization by introducing appropriate chemical moieties along with charged species for enhancing anisotropy related properties of these synthetic materials. ${ }^{7}$

In order to contain the inherent toxicity of these newer synthetic nanomaterials within safe limits, using phytochemicals based nanoparticulate species in place of more toxic inorganic and organic species is being perceived as a better route of synthesis. These are providing green routes of material synthesis dependent on employing inexhaustible resources available from the Nature. ${ }^{8}$ Current developments taking place in printing technology along with nano

\author{
Volume I Issue 4 - 2017
}

\author{
Ahmad S \\ Confederation of Indian Industry, India
}

Correspondence: S Ahmad, Confederation of Indian Industry, 508, Brentwood Tower, Eros Garden, Charmwood Village, Faridabad, Haryana, India, Tel 009I-9818I37484, Email drsahmad@email.com

Received: September 29, 2017 | Published: October 03, 2017

inks prepared out of engineered nanomaterials are emerging in form of an anticipated additive technology in contrast to the conventional subtractive microelectronics technology (i.e. comprising of thin film coatings, patterning by lithographic processing combined with wet/ dry etchings) to reduce the production costs of the miniaturized active devices, sensors, actuators, and their organizational integration in the form of intelligent subsystems for their immediate applications in developing Internet of Things (IoT) based strategies in various socio-economic sectors for successful implementation of intelligent decision makings. Adding various kinds of papers, plastics, and technical textiles as substrates in printing such types of affordable intelligent subsystems to gather data for interfacing to the cloud hub will certainly make the entire effort more environments friendly by using renewable resources than those scarce ones already explored in the recent past. ${ }^{9}$

The industrial scale production of intelligent subsystems and modules appears within reach for their widespread uses in upcoming IoT applications, where a trillion devices are projected to be in the field use by $2020 .^{10}$ It is also noteworthy that these printed functional modules will possibly be energized using on-site energy harvesting devices particularly with the help of perovskite halide materials. ${ }^{11}$ Further efforts made in understanding the controlled syntheses of these perovskite halide based mono/multiple layers will usher in an altogether new concept in producing solar photovoltaic devices with comparable power conversion efficiency of silicon. Perhaps the major drawback of material generated device instabilities will also be handled better with the help of 2D nanomaterials in near future. ${ }^{12}$

\section{Acknowledgements}

None.

\section{Conflict of interest}

The author declares no conflict of interest.

\section{References}

1. Ahmad S. Materials by design-prospects and challenges. Indian J Engineering \& Materials Sciences. 2005;12(4):299-316.

2. Ahmad S. Band-structure-engineered materials synthesis-nanocrystals and hierarchical superstructures current status and future trend. Int J Material Science. 2016;6(1):12226. 
3. Talapin DV, Lee JS, Kovalenko MV, et al. Prospects of colloidal nano crystals for electronic and optoelectronic applications. Chem Rev. 2010;110(1):389-458.

4. Xu Q, Zhang W, Dong C, et al. Biomimetic self-cleaning surfaces: synthesis, mechanism and applications. J R Soc Interface. 2016;13(122):112 .

5. Kumar D, Ahmad S. Green intelligent nanomaterials by design (Using Nanoparticulate/2D-materials building blocks) current developments and future trends; 2017.

6. Ahmad S. Band Gap engineering of hybrid 2-D nanomaterials some recent developments. MOJ Poly Sci. 2017;1(2):00010.

7. Lin Z, Mc Creary A, Briggs N, et al. 2D materials advances: from large scale synthesis and controlled heterostructures to improved characterization techniques, defects and applications. 2D Mater. 2016;3(4):042001.
8. Ahmad S, Hashim U. Nano-herbals in human healthcare: a proposed research and development-roadmap I, and II. ASEAN J Science and Technology for Development. 2012;29(1):55-75.

9. Moinudeen GK, Ahmad F, Kumar D, et al. IoT applications in future foreseen guided by engineered nanomaterials and printed intelligence technologies a technology Review. Int J Internet of Things. 2017;6(3):106148.

10. New production method for $2 D$ materials could lead to smarter devices. USA: National physics laboratory; 2017.

11. Ahmad S. An affordable green energy source - Evolving through current developments of organic, dye sensitized, and perovskite solar cells. Int $J$ Green Energy. 2016;13(9):859-906.

12. Xiaojun Qin, Zhao Z, Wang Y, et al. Recent progress in stability of perovskite solar cells. J Semicond. 2017;38(1):011002. 